\title{
Enfermedad de Orina Olor a Jarabe de Arce: presentación de caso clínico
}

\author{
Maple Syrup Urine Disease: A case report \\ Tania Soledad Licona Rivera, * Linda Yajeira Banegas Pineda. **
}

\section{RESUMEN}

Antecedentes: La enfermedad de orina olor a jarabe de arce (EOJA) es un error congénito del metabolismo de herencia autosómica recesiva, causado por la actividad defectuosa del complejo enzimático deshidrogenasa de a-cetoácidos, ocasionando que los aminoácidos de cadena ramificada; valina, leucina e isoleucina no puedan catabolizarse completamente.

Se trata de lactante menor, tres meses de edad, con antecedente de vómitos frecuentes $y$ rechazo a la alimentación desde la primera semana de vida, tratado por alergia a la proteína de la leche de vaca y reflujo gastroesofágico grado IV, con varios cambios de formula en su alimentación. Trasladado al Instituto Hondureño del Seguro Social, Hospital Regional del Norte (IHSS-HRN) con historia de cinco días de tos, fiebre y aproximadamente nueve horas de dificultad respiratoria. Tres horas más tarde presenta convulsiones tónicas y choque, por lo que se trasladado a sala de cuidados intensivos pediátricos, acoplándose a ventilador mecánico. Laboratorialmente: acidosis metabólica persistente que se logró controlar a las 48 horas, Anión Gap: 17, cetonuria, IRM con importante atrofia cortical. Se encontró elevación de los metabolitos de aminoácidos de cadena ramificada; 2-OH isovalerico, 2- $\mathrm{OH}$ isocaproico, 2-ceto-3 methylvalerico, 2 cetoisocaproico consistentes con EOJA y elevación del ácido láctico y alfa cetoglutarato; que podrían indicar defectos en la subunidad E3 de la enzima

*Pediatra egresada del Postgrado de Pediatría, UNAH-VS.

**Pediatra intensivista, Instituto Hondureño de Seguridad Social.

Dirigir correspondencia a:tania_licona@yahoo.com.mx

Recibido: 13 de agosto 2014

Aprobado: 04 de abril 2015 deshidrogenasa. Conclusiones: Los errores innatos del metabolismo son más frecuentemente diagnosticados cada día, y deben sospecharse en los niños con vómitos frecuentes.

\section{PALABRAS CLAVE}

Aminoácidos de Cadena Ramificada, Cetosis, Enfermedad de la Orina de Jarabe de Arce.

\section{ABSTRACT}

The maple syrup urine disease (MSUD) is an inborn error of the metabolism of autosomal recessive inheritance. It is caused by defective activity of the a-keto acids dehydrogenase enzyme complex; therefore, branched chain amino acids like valine, leucine and isoleucine cannot be fully catabolized.

A three month old infant is presented. The child had a history of frequent vomiting and refusal to feed since the first week of life. The child was treated as a cow milk protein allergy and grade IV gastroesophageal reflux with several changes of formula according to the diagnosis. Currently, she was admitted at the hospital with history of five days of cough, fever and approximately nine hours of respiratory distress. Three hours later the child presents tonic seizures and shock. She was then transferred tot he pediatric intensive care unit, and she was attached to mechanical ventilator. The patient presented ketonuria and persistent metabolic acidosis with elevated anion gap (17), which resolved in 48 hours. IRM showed important cortical atrophy. Elevated metabolites levels of branched chain amino acids were found; 2-OH isovaleric, 2-OH isicaproico, 2-keto-3-methylvalerico, 2 ketoisocaproico, all of 
them consistent with EOJA and elevation of lactic acid and Alpha-ketoglutarate; that it could indicate defects in the E3 enzyme dehydrogenase subunit. Inborn errors of metabolism are most frequently diagnosed every day, and they should be suspected in children with frequent vomiting.

\section{KEY WORD}

Amino Acids, Branched-Chain, ketosis, Maple Syrup Urine Disease.

\section{INTRODUCCIÓN}

Los errores innatos del metabolismo (EIM) son un grupo amplio de enfermedades "entre las cuales se conocen más de 500," con una prevalencia global de $1 / 600$ recién nacidos vivos. ${ }^{(1)}$ La mayoría se manifiesta en la edad pediátrica desde las primeras horas de vida o hasta en la adolescencia, con síntomas y signos similares a otras patologías; no reconocerlas conduce a secuelas importantes siendo las más frecuentes: desnutrición, convulsiones y retardo mental. $^{(2)}$

La enfermedad de orina olor a jarabe de arce, también conocida como cetoaciduria de cadena ramificada o leucinosis es un EIM, de herencia autosómica recesiva, causado por la actividad defectuosa del complejo enzimático deshidrogenasa de a-cetoácidos, ocasionando que los aminoácidos de cadena ramificada; valina, leucina e isoleucina (VIL) no puedan catabolizarse completamente, produciendo un bloqueo metabólico que ocasiona el acúmulo de sus metabolitos; a-cetoisocaproico a-cetometilvalérico a-cetoisovalérico, que son los responsables del efecto tóxico al cerebro. Fue reportada por primera vez en 1954 por Menkes quien detectó tres casos en una familia y se describe como "enfermedad neurodegenerativa" de inicio neonatal y con desenlace letal hacia el tercer mes de vida; los afectados presentan olor particular en la orina, semejante al jarabe de arce usado como alimento. ${ }^{(3,4)}$

La leucina, isoleucina y la valina son aminoácidos esenciales de cadena ramificada (AACR), sus funciones biológicas comprenden la síntesis de proteínas, de ácidos grasos y colesterol, la restauración del balance nitrogenado y un freno del catabolismo que se observa en condiciones como sepsis y politraumatismo. Su metabolismo incluye incorporación a proteínas y procesos de degradación oxidativa en las mitocondrias, siendo importantes como fuente alterna de energía en el musculo, cerebro, riñones, corazón y en los tejidos grasos. Los ácidos a-cetoisocaproico, a-cetometilisovalérico y a-cetoisovalérico derivados de la leucina, de la isoleucina y de la valina, son alfa cetoácidos ramificados que se metabolizan en el hígado para producir cuerpos cetónicos y derivados coenzima $A^{(3)}$

La prevalencia varía según la población, va desde 1:290.000 recién nacidos (RN) en la población anglosajona, a 1:200 RN en la población Menonita de los Estados Unidos, debido a la alta consanguinidad en este grupo étnico. ${ }^{(5,6)}$ En la actualidad, el estándar de oro para la detección oportuna de esta enfermedad es el Tamiz Neonatal Ampliado (TNA) por espectrometría de masas en tándem (MS/MS), también se puede realizar el análisis de aminoácidos plasmáticos mediante cromatografía de líquidos de alta resolución(CLAR) que muestran el incremento en las concentraciones de leucina, valina e isoleucina, pudiendo en ocasiones observarse un decremento de la concentración de alanina. ${ }^{(7)}$

El complejo enzimático deshidrogenasa está compuesto por 3 unidades: la E1 conformada por dos subunidades: E1a (cromosoma 19q13.1-q13.2) y la E1b (cromosoma 6p21-p22) dependiente de tiamina. La unidad E2 ubicada en el cromosoma 1 p31 y la unidad E3 mapeada en el cromosoma 7q31-q32. ${ }^{(1,2)}$

Las manifestaciones dependerán de la clasificación clinica:

a) CLÁSICA O NEONATAL: se manifiesta entre el $5^{\circ}$ y $10^{\circ}$ día de vida en un RN de término, con rechazo de la alimentación, somnolencia y 
coma. Posteriormente aparece falta de regulación neurovegetativa, distres respiratorio, apnea, bradicardia e hipotermia.

Tienen un olor característico a azúcar quemada o jarabe de arce en piel y orina. El diagnóstico se confirma al encontrar elevación de los aminoácidos VIL en sangre y orina y por la presencia del metabolito L-aloisoleucina, patognomónico en esta patología. Recientemente se ha demostrado que el ácido a-cetoisocaproico derivado de la leucina causa apoptosis tanto en células gliales como neuronales, lo que podría explicar el daño neurológico producido por la descompensación metabólica en cualquier momento de la vida.

b) INTERMITENTE: se manifiesta en etapa preescolar o en la adolescencia, con episodios recurrentes desencadenados por infecciones e ingesta alta de proteínas. Los síntomas más importantes son letargia, vómitos, ataxia, deshidratación, cetoacidosis e hipoglicemia. La elevación de los aminoácidos VIL en suero se detecta sólo en los períodos de descompensación.

c) INTERMEDIA: presentan anorexia persistente, vómitos crónicos, retardo de crecimiento y retardo mental. Los aminoácidos VIL están elevados permanentemente pero en menor grado que la forma clásica. Estos pacientes son diagnosticados entre el $5^{\circ}$ mes y los 7 años por retardo en el desarrollo y por un olor especial.

d) RESPONDEDORA A TIAMINA: es similar a la forma intermedia, y es dependiente de tiamina (suplementación con 10 y 1000 mg/día). No se ha descrito ningún paciente tratado exclusivamente con tiamina, requiriéndose la complementación con la dieta restringida en los aminoácidos ramificados.

e) DEFICIENCIA DE DIHIDROLIPOIL DESHIDROGENASA E3: es poco frecuente y el cuadro clínico es similar a la forma intermedia, con aumento de los aminoácidos VIL, ácido láctico, ácido pirúvico y a-cetoglutárico. Entre los 2 y 6 meses se produce una importante acidosis láctica, con deterioro neurológico, hipotonía y movimientos anormales. ${ }^{(6)}$

Para su tratamiento es necesario diferenciar la fase en la que se encuentra el paciente (fase aguda o fase de mantenimiento). Los objetivos en la fase de descompensación metabólica aguda se basan en tres puntos: eliminar los metabolitos tóxicos, soporte nutricional y conseguir anabolismo. El primer aspecto se consigue con diálisis peritoneal, hemodiálisis o exanguinotransfusión. En la fase de mantenimiento el objetivo del tratamiento es mantener las concentraciones plasmáticas de los aminoácidos VIL en los niveles próximos posible a los valores normales. La leucina es el que se encuentra en mayor proporción en los alimentos naturales y es el más neurotóxico, por lo que su estricta monitorización es fundamental, debiéndose mantener su concentración plasmática entre 2-6 mg/dl. Para alcanzar las necesidades calóricas se añaden alimentos libres, totalmente exentos de AACR, como aceites (oliva, maíz, girasol,) hay que realizar controles analíticos nutricionales periódicamente, para prevenir $y / o$ corregir posibles deficiencias habiendo sido descritas anemias megaloblásticas por deficiencia de ácido fólico, deficiencia de selenio, carnitina, etc. Se recomienda suplemento de por lo menos 50 mg/día de tiamina, aunque deben tratarse según tolerancia individual de cada paciente y dosis farmacológicas de tiamina, aunque estas dosis oscilan en un amplio margen que va de 100 a 1.000 mg/día. ${ }^{(8)}$

Las mejoras en el entendimiento, monitoreo, manejo oportuno y adecuado de los pacientes permiten disminuir la morbilidad y mortalidad por EOJA. La edad de diagnóstico y el curso de la enfermedad siguen siendo factores independientes, que pueden afectar el pronóstico a largo plazo. El riesgo de presentar complicaciones por descompensación aguda sigue siendo elevado hasta los seis años de vida, tiempo en el que el organismo es capaz de tolerar mejor el 
estrés. Se ha observado que los pacientes que presentan pobre desarrollo intelectual en general, ya cursaban con otras secuelas neurológicas. ${ }^{(3)}$

El objetivo de publicar este caso clínico, es dar a conocer que en un niño con vómitos persistentes, debe considerarse dentro del diagnóstico diferencial los errores innatos del metabolismo, su diagnóstico oportuno conlleva a una disminución importante en la morbimortalidad del paciente pediátrico.

\section{CASO CLINICO}

Lactante masculino de tres meses de edad, producto de segundo embarazo que curso sin complicaciones, madre de 24 años de edad, ama de casa, casada, procedente de Puerto Cortés, nace de término vía cesárea sin complicaciones, con peso de $2.7 \mathrm{~kg}$. Con antecedente de vómitos frecuentes y rechazo a la alimentación desde la primera semana de vida, tratado por alergia a la proteína de la leche de vaca y reflujo gastroesofágico grado IV (según resultado de serie esofagogastroduodenal realizado a los dos meses de edad), evaluado por diferentes médicos generales, especialista y subespecialistas; alimentado con diferentes fórmulas incluyendo leche de soya, hidrolizados y finalmente leche de arroz desde la última semana, siendo la única que toleraba. Referido al I.H.S.S. con historia de cinco días de tos húmeda, no emetizante ni cianotizante y fiebre, tratado con claritromicina y ambroxol, llevado al Hospital de área de Puerto Cortés por dificultad respiratoria de aproximadamente 9 horas de evolución, siendo referido al IHSS- HRN con puntaje de 4, en la escala de Wood Downes. Tres horas posteriores a su ingreso presenta frialdad distal, llenado capilar de 3 segundos y convulsiones tónicas de difícil manejo, por lo que fue trasladado a la sala de cuidados intensivos pediátricos y se acopló a ventilador mecánico.

Ingresa a UCIP del I.H.S.S. con diagnóstico de bronquiolitis más estatus convulsivo en estudio, ante la fuerte sospecha de neuroinfección, se realiza punción lumbar encontrando análisis de líquido cefalorraquídeo normal. Gases arteriales con acidosis metabólica severa compensada (ph: 7.36, Pco2: 10.1, HCO3: 5.8, EB:-16.7) tratado con bicarbonato en los líquidos y se logró controlar a las 48 horas, Anión Gap: 17, cetonuria $(+++)$, hemograma con leve granulocitosis, PCR negativa, prueba rápida de influenza negativa, TAC cerebral normal, IRM con importante atrofia cortical de predominio frontal, y restricción de la difusión en ganglios basales y sustancia blanca en T2. Fig. No 1.

Figura No. 1: Imagen de la IRM del paciente.

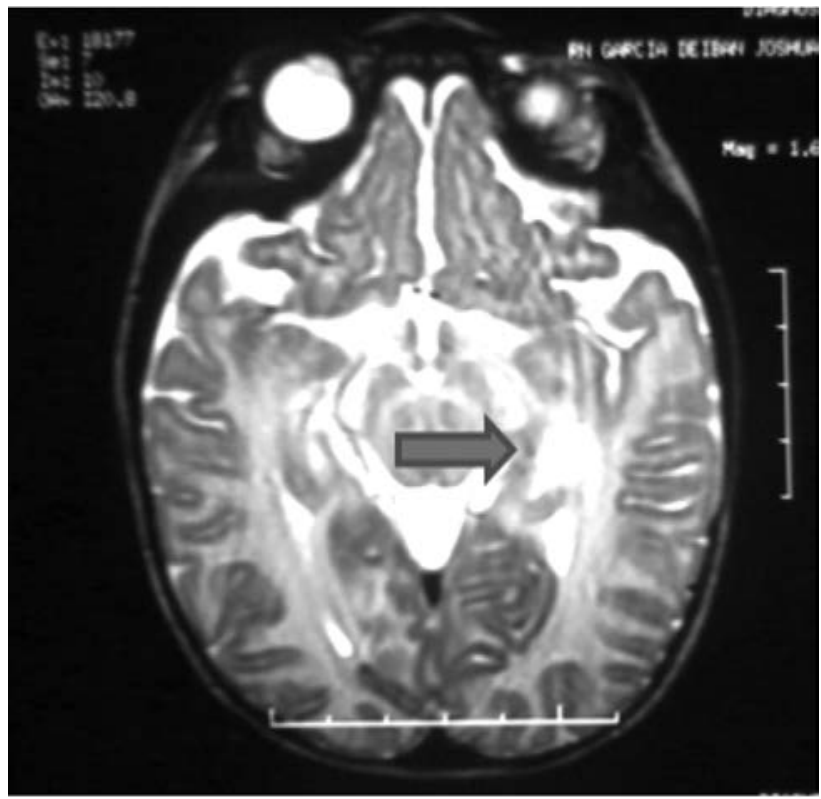

Se observa la restricción de la difusión en ganglios basales y sustancia blanca en $\mathrm{T} 2$.

Ante la persistencia del cuadro emético y convulsivo, se consideró la posibilidad de errores innatos del metabolismo, sin embargo el resultados del Tamiz metabólico fue negativo. Estuvo ingresado durante 13 días, posteriormente egresó con formula de arroz que era la única que toleraba más suplementos como L-carnitina, sulfato ferroso, zinc. Se indica cita con endocrinóloga pediatra del IHSS en Tegucigalpa quien envía muestras de orina al laboratorio Greenwood Genetic Center, de Estados Unidos, encontrando elevación de los metabolitos de los aminoácidos de cadena ramificada: 
$2 \mathrm{OH}$ - isovaleric, $2 \mathrm{OH}$-isocaproico, 2- ceto-3methylvalerico, 2-cetoisocaproico consistentes con Enfermedad de orina olor a jarabe de arce, simultáneamente se encontró elevación del ácido láctico y a cetoglutarato; que podrían indicar defectos en la subunidad E3 de la enzima deshidrogenasa.

También se realizó cromatografía liquida de alta definición (HPLC), encontrando los aminoácidos esenciales VIL elevados. Ver Tabla No 1.

Tabla No. 1: Resultados de cromatografía liquida de alta definición, del paciente.

\begin{tabular}{lcc}
\hline AA ESCENCIALES & PACIENTE & NORMAL \\
\hline Valina & $457 \mu \mathrm{mol} / \mathrm{L}$ & $250-400 \mu \mathrm{mol} / \mathrm{L}$ \\
Leucina & $739 \mu \mathrm{mol} / \mathrm{L}$ & $150-250 \mu \mathrm{mol} / \mathrm{L}$ \\
Isoleucina & $308 \mu \mathrm{mol} / \mathrm{L}$ & $150-250 \mu \mathrm{mol} / \mathrm{L}$ \\
\hline
\end{tabular}

Nótese que todos los aminoácidos están elevados, predominantemente la leucina.

A los cinco meses, pesaba $5 \mathrm{Kg}$, siendo lo ideal para su edad $6.5 \mathrm{~kg}$, con irritabilidad. Para continuar su tratamiento, se trasladó con su madre, a vivir con familia que apoyan a personas con dicha enfermedad (Familia Menonita) en Estados Unidos y continuar su atención medica en Clínica especial de Lancaster Pensilvania.

El tratamiento de mantenimiento que recibe en Honduras, consiste en alimentación con fórmula especial traída desde E.E.UU. SMUD-JR, recibiendo 100-110 cal/kg/día.

Posteriormente presento dermatitis secundaria a deficiencia de VIL. Figura No 3.

Pese a que los niveles séricos de estos tres aminoacidos se encuentran elevados en esta patología, siempre deben recibir suplemento dietético de los mismos, en este caso el $25 \%$ de los basales; Leucina 55-65 mg/kg-día, Isoleucina 35-45 mg/kg/día, Valina 50-60 mg/kg/día. Los aminoácidos valina e isoleucina en tabletas de $500 \mathrm{mg}$ son mezclados (2tabletas $=1000 \mathrm{mg}+$ $100 \mathrm{ml}$ de agua) para obtener $10 \mathrm{mg} / \mathrm{ml}$, y esta es la forma de mantener los niveles plasmáticos adecuados de VAL.

\section{Figura No. 3: Paciente con dermatitis por deficiencia de VIL.}

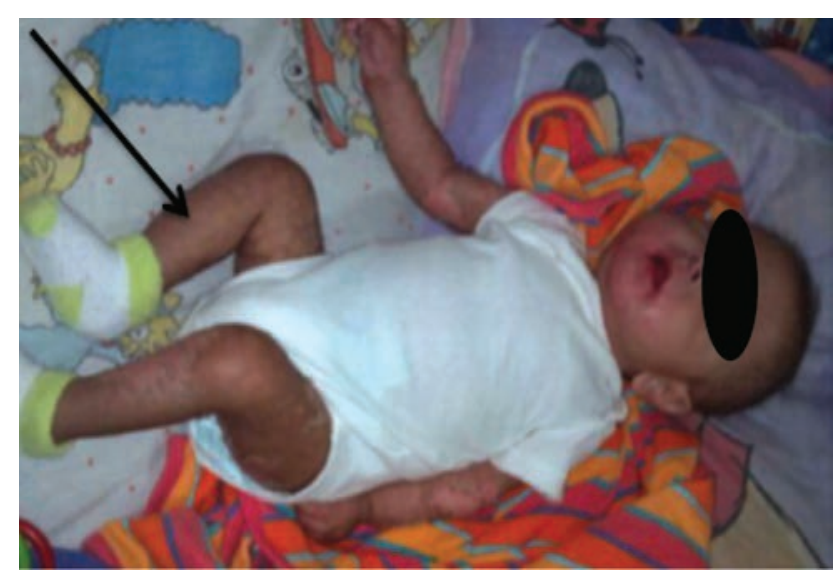

Nótese las áreas de descamación en las extremidades.

Semanalmente se realizan controles de HPLC en papel filtro, para asegurar un rango normal de VAL.

\section{DISCUSION}

La Enfermedad de orina, olor a Jarabe de Arce, es un error innato del metabolismo que de no tratarse tempranamente, ocasiona graves secuelas neurológicas. En nuestro País, aún estamos en pañales en cuanto al diagnóstico y manejo de esta enfermedad, afortunadamente la incidencia es baja siendo este uno de los primeros casos en diagnosticarse; no dejando de ser útil todos los avances que de aquí en adelante se lleven a cabo para mejorar en la pesquisa de estas enfermedades. El diagnóstico y tratamiento de nuestro paciente, fue posible con la ayuda de la Clínica Especial para niños con esta enfermedad en Pensilvania, que fue creada en 1989. Antes de esta fecha, casi la mitad de los niños morían por hernia cerebral y muchos de los que sobrevivieron presentaron secuelas neurológicas permanentes. ${ }^{(9)}$

La experiencia de Chile en el diagnóstico y 
seguimiento de pacientes con EOJA, comienza también en 1989, para el año 2005 se habían diagnosticado 31 niños con esta patología con un promedio de edad al diagnóstico de 12.8 días, de los cuales 30 fueron diagnosticados por una descompensación aguda en el período de recién nacido. ${ }^{(5)}$ Similar a lo que sucedió con nuestro paciente, que fue diagnosticado debido a su descompensación, pero sus estudios tardaron en realizarse por no haber disponibilidad en nuestro país.

La dermatitis, la pérdida de peso y la irritabilidad que presento el paciente se explica por el déficit de isoleucina, pues el déficit prolongado de isoleucina (menor de $50 \mu \mathrm{M} / \mathrm{L}$ ) produce pérdida o detención de ganancia de peso, decoloración de la mucosa bucal, fisuras en la comisura de labios, temblor de extremidades y descamación de la piel. El déficit de valina (menor de $150 \mu \mathrm{M} / \mathrm{L}$ en suero) ocasiona anorexia, mareos, irritabilidad, llanto persistente, pérdida de peso, disminución de albúmina plasmática. Los síntomas neurológicos como ataxia y dificultad para mantener la concentración en tareas específicas, pueden ser los primeros síntomas de exceso de leucina. ${ }^{(5)}$

En el momento que se le realizó el diagnóstico a nuestro paciente, presentaba niveles de leucina de $739 \mu \mathrm{mol} / \mathrm{L}$, lo cual es muy elevado y de todos los aminoácidos de cadena ramificada es el más neurotóxico, por lo cual se recomienda mantenerlo en rangos normales para obtener buen desarrollo cognitivo-psicomotor. En los últimos años, autores como Hoffmann et al. ${ }^{(10)}$ recomiendan en los niños lactantes y preescolares que las concentraciones de leucina no excedan de $200 \mu \mathrm{mol} / \mathrm{l}$ para conseguir el mejor desarrollo intelectual posible.

Con los estudios genéticos que se realizó a nuestro paciente, se concluyó que la variante de la enfermedad que presenta coincide con defectos de la subunidad E3 de la enzima deshidrogenasa, porque además de estar elevados los metabolitos intermedios del metabolismo de los VIL, simultáneamente presentaba elevación del ácido láctico y alfa cetoglutarato. Esta es una forma poco frecuente con aumento de los aminoácidos VIL, ácido láctico, ácido pirúvico y a-cetoglutárico. Entre los 2 y 6 meses se produce una importante acidosis láctica, con deterioro neurológico, hipotonía y movimientos anormales. ${ }^{(5,11)}$ Hecho que coincide con la edad en que nuestro paciente exacerbo su sintomatología.

Es de hacer notar que el estudio de cromatografía liquida de alta definición es de aproximadamente \$10.00 por cada aminoácido (alrededor de 200 lempiras) y sin embargo no está disponible en nuestro país, por lo cual es más difícil realizar un diagnóstico de este tipo.

En conclusión, como parte de la pesquisa de las enfermedades congénitas del metabolismo, se debe incluir asesoría prenatal, donde el ginecólogo trasmita a los padres un mensaje general del estas enfermedades. El pediatra o neonatólogo deberá solicitar a todo recién nacido un tamiz neonatal, siempre teniendo a su cargo la tarea de convencer a los padres de invertir en la realización de este examen.

Se recomienda a las autoridades del I.H.S.S. y de la Secretaría de Salud, destinar presupuesto para realizar tamiz neonatal ampliado a todo recién nacido en las primeras 72 horas de vida.

Agradecimiento: A la Dra. Lesby Espinoza, endocrinóloga pediatra del Hospital Materno Infantil e Instituto Hondureño del Seguro Social de Tegucigalpa. Médico tratante, quien gestionó en el extranjero para que se realizaran las pruebas necesarias para el diagnóstico definitivo, y por brindarnos los resultados de dichas pruebas. 


\section{BIBLIOGRAFÍA}

1. Martín S. M., Legarda T. M., Dalmau S. J. Errores innatos del metabolismo: aproximación diagnóstica enAtención Primaria. BolPediatr 2007; 47: 111-115.

2. Raimann BE. Diagnóstico de errores innatos del metabolismo. Rev. chil. pediatr. [revista en la Internet]. [citado 2013 Ago 01]. Disponible en: http://www.scielo.cl/scielo. php? script=sci_arttext\&pid=S0370-41062008 000700014\&lng=es. http: //dx.doi.org/10. 4067/S0370-41062008000 700014.

3. Gómez CJF, Espinoza GE, Barrera LA, Echeverry OY. Enfermedad de orina en jarabe de arce: Mejoria clínica asociada a detección precoz y manejo oportuno. Reporte de caso y revisión de literatura. Rev. Fac. Med [serial on the Internet]. 2008 June [cited 2013 Aug 30] ; 16(1): 99-105. Available from: http://www.scielo.org.co/scielo.php? script $=$ sci_arttext\&pid=S0121-52562008 000100013\&lng=en.

4. Strauss KA, Wardley B, Robinson D, Hendrickson Ch, Rider NL, et al. Classical maple Syrup urine disease and brain development: principles of management and formula design. Molecular Genetics and Metabolism 99 (2010);333-45.

5. Cornejo E Verónica, Raimann B Erna. Actualización en el tratamiento agudo y crónico de la enfermedad orina olor a jarabe de arce de presentación neonatal. Rev. chil. nutr. [revista en la Internet]. 2005 Dic [citado 2013 Ago 30] ; 32(3):200-206. Disponible en: http://www.scielo.cl/scielo.php? script=sci_arttext\&pid=S0717-7518 2005000300004\&lng=es. http://dx.doi. org/10.4067/S0717-75182005000300004.

6. Couse Pico M.L., Castiñeiras Ramos D.E.,
Bóveda Fontan M.D., Iglesias Rodriguez A.J., Cocho de Juan J.A., et. al. Avances en el diagnóstico y tratamiento de la enfermedad de jarabe de Arce, Experiencia en Galicia. An Pediatr. (Barc.). 2007; 67(4)337-43.

7. Ibarra G. I., Fernández L. C., Belmont M. L. Vela A. M. Elevada mortalidad y discapacidad en niños mexicanos con enfermedad de orina con olor a jarabe de arce (EOJA). [Citado 2013 Ago 01];GacMédMéx Vol. 143 No. 3, 2007. Disponible en: http://www. medigraphic.com/pdfs/gaceta/gm2007/gm073d.pdf

8. Luis Román D. A. de, Izaola Jáuregui O. Enfermedad de jarabe de arce: una entidad rara que debemos recordar. A propósito de su manejo dietético. An. Med. Interna (Madrid) [revista en la Internet]. 2005 Oct [citado 2013 Ago 30] ; 22(10):493-497. Disponible en: http://scielo.isciii.es/scielo.php? script=sci_arttext\&pid=S0212-7199 2005001000010\&lng=es. http://dx.doi.org/ 10.4321/S0212-71992005001000010.

9. Strauss K.A., Wardley B., Robinson D., Hendrickson CH., Puffenberger E. G. et. al. Classical maple syrup urine disease and brain development: Principles of management and formula design. Molecular Genetics and Metabolism 99 (2010) 333-345

10. Hoffmann B, Helbling Ch, Schadewaldt $P$, Wendel U. Impact of longitudinal plasma leucine levels on the intellectual outcome in patients with classic MSUD. Pediatr Res. 2006;59:17-20.

11. Kleopa KA, Raizen DM, Friedriech CA, Brown MJ, Bird SJ. Acute axonal neuropathy in Maple Syrup Urine Disease, MuscNer.2001; 24:284-287 